\title{
A study on Complications of ventriculoperitoneal shunt surgery in Bir Hospital, Kathmandu, Nepal
}

\author{
Karmacharya $B G,,^{1^{*}}$ Kumar $P^{2}$
}

${ }^{1}$ Manipal Teaching Hospital, Pokhara, Nepal, ${ }^{2}$ National Neurosurgical Referral Center, Bir Hospital, Kathmandu,Nepal

\section{*Corresponding Author: \\ Dr BalGopal Karmacharya MS, MCPS, FCPS \\ Manipal Teaching Hospital \\ Phulbari, Pokhara-33701, Nepal \\ Email:dr.balgopal@hotmail.com}

\section{Citation}

Karmacharya BG, Kumar P. A study on Complications of ventriculoperitoneal shunts surgery in Bir Hospital, Kathmandu, Nepal. Nepal Journal of Medical sciences 2012;1(2):119-22.

\begin{abstract}
Background: Ventriculoperitoneal shunt is one of the most commonly performed neurosurgical procedure, both on the elective and emergency basis. However this procedure is dreaded because of complications. There is lack of prospective studies on complications of shunt procedure. In this study, the indications for shunt, the types used and complications of ventriculoperitoneal shunts were studied.
\end{abstract}

Methods: This was a prospective study carried out in the national neurosurgical referral centre, Bir hospital, Kathmandu from April 2004 to March 2005.

Results: There were 109 ventriculoperitoneal shunt procedures during the study period. Among them 60 consecutive patients who fulfilled the inclusion criteria were enrolled for the study. There were 43 male and 17 female patients, with age ranging from 4 months to 75 years. Fourteen patients $(23.3 \%)$ developed complications which included shunt block, shunt infection, over drainage and shunt extrusion.

Conclusion: About one fourth all patients who underwent ventriculoperitoneal shunt surgery developed complications. Shunt block and infections were the major complications.

Keywords: Hydrocephalus; ventriculoperitoneal shunt; slit ventricle syndrome; Staphylococcus epidermidis

\section{Background:}

ydrocephalus literally means collection of water in the brain and is defined as enlargement of the ventricles with an associated increase in the volume of cerebrospinal fluid. ${ }^{1}$ Hydrocephalus results from various conditions that lead to over secretion, impaired absorption or obstruction to the pathways of the cerebrospinal fluid in the ventricular system or subarachnoid spaces.
Ventriculoperitoneal shunt is one of treatment options for treating hydrocephalus, both obstructive and communicating. It is also one of the most commonly performed neurosurgical procedures, both on the elective and emergency basis. Ventriculoperitoneal shunt procedures accounted for $14 \%$ of neurosurgical case load in a tertiary neurosurgical center. ${ }^{2}$

However it is associated with many potential complications which can be fatal. The complications are of the following 
three categories- mechanical failure of the device, functional failure due to overdrainage or underdrainage and infection of cerebrospinal fluid or shunt. ${ }^{3}$ Various factors including patient's age, sex, type of hydrocephalus, and duration of surgery, experience of operating surgeon and use of prophylactic antibiotics have been studied extensively that are thought to contribute to such complications..$^{4-13}$

\section{Methods:}

This was a prospective descriptive study of patients who underwent ventriculoperitoneal shunt for hydrocephalus due to various neurosurgical causes. The study was carried out in the National Neurosurgical Referral Centre (NNRC) in Bir Hospital which is the only tertiary level neurosurgical care centre in the government sector. Only the patients who had their first ventriculoperitoneal shunts were included in the study and excluded were the patients who underwent shunt revisions, external ventricular drain, ventriculoatrial and lumboperitoneal shunts and who did not complete two months follow up.

There were a total of 109 patients who had shunt procedures done during the study period. Among them 60 patients who fulfilled the inclusion criteria and consented to take part on the study were enrolled.

The patients were seen in the emergency room or the neurosurgical out-patient clinic. Information was collected regarding presenting complaints, past medical and surgical illnesses. The ventriculoperitoneal shunting was done electively or emergently based on clinical indication. After the surgery, the patients were followed up in the ward till discharge and then in the neurosurgical out patient clinic. Any complications were noted during the follow up period. The minimal follow up period was of eight weeks.

\section{Results:}

From 2004 April to 2005 March, 109 patients underwent ventriculoperitoneal shunt. Sixty patients of them who fulfilled the inclusion criteria were studied.

There were 43 male and 17 female patients with mean age of 12.7 years ( 4 months to $75 \mathrm{yrs}$ ). Twenty two patients $(36.6 \%)$ had obstructive hydrocephalus and 38 (63.3\%) patients had communicating hydrocephalus. Causes of hydrocephalus noted were as shown in table 1 .

Fifty five patients $(91.6 \%)$ had occipitoparietal burr hole and 5 patients $(8.3 \%)$ had frontal burr holes for the placement of ventriculoperitoneal shunt. Forty eight (80\%) patients had medium pressure shunt and 10 patients (16.6\%) with open fontanel had low pressure shunts placed. Two patients $(3.3 \%)$ who had large posterior fossae tumors with gross hydrocephalus had high pressure shunts for gradual decompression of the ventricles.

\section{Table 1: Causes of Hydrocephalus}

\begin{tabular}{lcc}
\multicolumn{1}{c}{ Causes } & Number & Percentage \\
\hline Brain tumors & 23 & 38.3 \\
Postmeningitic & 13 & 21.3 \\
Meningo/encephaloceles & 4 & 6.6 \\
Cerebellar hematoma & 4 & 6.6 \\
Acqueduct stenosis & 3 & 5 \\
Chiari malformation & 1 & 1.6 \\
Intraventricular hemorrhage & 3 & 5 \\
Neurocysticercosis & 2 & 3.3 \\
Others & 7 & 11.6 \\
\hline
\end{tabular}

Fourteen patients (23.3\%) developed complications during the follow up period of two months. Five patients (8.3\%) had shunt block, ventricular end in three patients and peritoneal end in two. Two patients (3.3\%) had overdrainage with symptomatic subdural hematoma needing evacuation and temporary occlusion of the shunt tube. Five patients $(8.3 \%)$ developed clinical shunt infection. Shunt complications were detected after a mean duration of 14.2 days (1-53 days).

\section{Discussion:}

The aim of the ventriculoperitoneal shunt is to establish a communication between the cerebrospinal fluid (ventricular end) and the drainage cavity (peritoneal end) where it is absorbed. It provides an alternative place for absorption of cerebrospinal fluid that is not absorbed within the cranial cavity. However, shunting exposes the patient to many potential complications, even several years afterwards. The complications can occur in the proximal ventricular end, along the shunt tract and at the distal peritoneal end. Studies have shown there might be many contributing factors like patient factors, procedure related factors and operating surgeon factors.

\section{Shunt infection}

Shunt infection remains a major cause of morbidity and 
mortality following cerebrospinal fluid shunts. Shunt infection occurs in $0.17-30 \%$ of patients. ${ }^{7,14}$ Infection can be localized to the shunt itself, meningeal sheaths, and peritoneum or along the shunt tract. Staphylococcus epidermidis causes approximately $60 \%$ of shunt infections, Staphylococcus aureus causes $30 \%$ and coliforms, propionebacteria and streptococci cause the remainder. ${ }^{6}$

Systemic and intrashunt antibiotics, iodine impregnated transparent surgical drapes, covering incisions with betadine soaked sponges may reduce shunt infections. In our study, five patients $(8.3 \%)$ developed shunt infection. Among five patients, only two patients had organism grown on cultures, one Staphylococcus aureus and the other Staphylococcus epidermidis.

\section{Shunt malfunction}

Shunt obstruction can occur anywhere along the shunt course and anytime during their lifetime. Shunt malfunction can be due to shunt infection which may be clinically undetectable. Negative cultures of cerebrospinal fluid taps do not exclude shunt infection in malfunctioning shunts.

Shunt malfunction can be also due to occlusion the ventricular end by choroid plexus, tumor growth or intraventricular blood. Similarly malfunction may be due to shunt disconnection, valve malfunction or kinking. In the peritoneal end the shunt malfunction is attributed to formation of pseudocyst and low grade peritonitis. In this study five patients had shunt malfunction. Among them the ventricular end was blocked in three patients and the peritoneal end was blocked in two patients.

\section{Shunt malposition}

Proper placement of ventricular and peritoneal ends is essential for proper functioning of the shunt. Ventricular end placement under ultrasound, endoscope or stereotactic guidance reduces such malposition. In one of our patients, ventricular end was malpositioned. Repositioning of the ventricular end was done.

\section{Shunt extrusion}

Shunt extrusion can occur anywhere along the catheter pathway. It can get extruded through scalp, abdominal wall, ${ }^{15}$ scrotum, anus or vagina. One patient in our study had distal end extrusion through the abdominal wall.

\section{Shunt overdrainage}

Subdural hygromas or hematomas may develop after shunting with very large ventricles and thin cerebral cortical mantles. Similarly overdrainage of cerebrospinal fluid can lead to slit ventricle syndrome and intractable low pressure headache. If there is formation of symptomatic subdural hematoma, it should be evacuated with temporary occlusion of the shunt tube. Two of our patients developed subdural hematomas.

\section{Conclusion:}

In our study about one fourth of all patients developed shunt complications. However shunt infection rate was comparable with other studies. This was a small prospective study. Further large studies are required to know about the long term complications of such shunt procedures.

Though most of the shunt infections occur in first two months, patients should be followed up for a long time as the complications especially shunt malfunction can develop even after several years. Shunt registry should be maintained by all treating neurosurgeons and there should be free communication between neurosurgeons as such patients may be seen by different surgeons.

\section{Conflict of interest: none}

\section{References:}

1. Contran RS, Kumar V, Robbins SL. Robbins Pathological Basis of Diseases. Philadelphia: WB Saunders, 1994.

2. Mukhida K, Sharma MR, Shilpakar SK. Management of hydrocephalus with ventriculoperitoneal shunt: Review of 274 cases. Nepal Journal of Neurosciences 2004;1:104-12.

3. Wilkins RH, Rengachary SS. Neurosurgery. New York: Mc Grow-Hill, 1996.

4. Dallacasa P, Dappoza A, Gallasi E, et al. Cerebrospinal fluid shunt infection in infants. Childs Nerv Syst 1995;11:643-9

5. Casey ATH, Kummings EJ, Kleinlingtebeld AD, et al. The long term outlook for hydrocephalus in children: A ten year cohort study of 155 patients. Paediatr Neurosurg 1997;27:63-70.

6. Pople IK, Bayston R, Hayward RD. Infection of cerebrospinal fluid shunts in infants: A study of etiological factors. J Neurosurg 1992;77:29-36.

7. Ammirati M, Raimondi AJ. Cerebrospinal fluid shunt infection in children. A study on the relationship between etiology of hydrocephalus, age at the time of 
Original Article | Karmacharya BG et al. complications of Ventriculoperitoneal shunts

shunt placement and infection rate. Childs Nerv Syst 1987;3:106-9.

8. Greibel R, Khan M, Tan L. Cerebrospinal fluid shunt complications: An analysis of contributing factors. Childs Nerv Syst 1985;1:77-80.

9. Tuli S, Drake J, Lawless J, et al. Risk factors for repeated cerebrospinal shunt failures in pediatric patients with hydrocephalus. Childs Nerv Syst. 2003;19:286-91.

10. Piatt JH Jr, Carlson CV. A search for determinants of cerebrospinal fluid shunt survival: retrospective analysis of a 14- yr institutional experience. J Paediatr Neurosurg 1993;19:233-41.

11. Lund-Johansen M, Swendsen F, Wester K. Shunt failures and complications in adults as related to shunt type, diagnosis and experience of surgeon. Neurosurgery 1994;35:839-44.
12. Del Bigio MR. Epidemiological and direct economic impact of hydrocephalus: A community based study. Can J Neurol Sci 1998;25:123-6.

13. Langley JM, Le Blanc JC, Drake J, et al. Efficacy of antimicrobial prophylaxis in placement of cerebrospinal fluid shunts: Metaanalysis. Clin Infect Dis 1993;17:98-3.

14. Kulkarni AV, Drake JM, Lamberti-Pascilli R. Cerebrospinal fluid shunt infection: a prospective study of risk factors. J Neurosurg 2001;94:195-21.

15. Chan Y, Datta NN, Chan KY, et al. Extrusion of the peritoneal catheter of a V-P shunt system through a gastrostomy wound. Surg Neurol 2003;60:68-9. 